\title{
Fuchs' Adenoma of the Choroid Simulating a Choroidal Hemangioma
}

\author{
Lubna Razzaq ${ }^{a}$ Marina Marinkovic ${ }^{a} \quad$ Wouter Swart $^{a}$ \\ Sjoerd G. van Duinen $^{b}$ Jan E.E. Keunen ${ }^{c}$ \\ Gregorius P.M. Luyten ${ }^{\text {a }}$ \\ Departments of ${ }^{a}$ Ophthalmology and ${ }^{b}$ Pathology, Leiden University \\ Medical Center (LUMC), Leiden, and 'Department of Ophthalmology, \\ Nijmegen University Medical Center, Nijmegen, The Netherlands
}

\section{Key Words}

Fuchs' adenoma · Ciliary body · Choroid · Hemangioma

\begin{abstract}
We report the case of a 54-year-old female who was referred to us with an amelanotic mass on the posterior pole of the left eye involving the macula. Fundus fluorescein angiography revealed a hyperfluorescent choroidal mass. Indocyanine green chorioangiography revealed a hypofluorescent choroidal lesion with hyperfluorescent margins. B-scan ultrasonography showed a choroidal mass with moderate reflectivity. Choroidal biopsy was performed, which revealed the diagnosis of Fuchs' adenoma.
\end{abstract}

\section{Introduction}

Fuchs' adenoma is a benign tumor, which develops at the pars plicata of the ciliary body. It is believed to be a reactive age-related proliferation of the nonpigmented ciliary epithelium; hence it is called age-related hyperplasia of the nonpigmented ciliary epithelium [1-4]. These are extremely common lesions, found in 20-31\% of eyes in two post mortem series, yet rarely encountered on clinical examination [2]. Fuchs' adenomas are benign but can rarely cause iris protrusion, shallowing of the anterior chamber, or glaucoma and have also been mistaken as iris or ciliary body melanomas $[3,5]$. We here report a case of choroidal Fuchs' adenoma in a middle-aged female, presenting as a mass at the posterior pole, and confirmed on biopsy. 


\section{Case Report}

A 54-year-old female was referred to our department with the possible diagnosis of choroidal hemangioma. She had had visual complaints in her left eye for 18 months. The patient had already received three sessions of photodynamic therapy (PDT), but there was no improvement and she was referred to our clinic. At that time, visual acuity was 20/60 in her left eye. Slit lamp examination of the anterior segment was normal. Non-contact lens fundoscopy revealed an amelanotic mass in the posterior pole, involving the macula and extending up to the superior temporal vascular arcade. The mass had some secondary changes and pigmentation in the inferior part and at the margins of the lesion (fig. 1a). Examination of the right eye was normal. Fluorescein angiography revealed a hyperfluorescent mass with early filling but no pinpoint leakage. Indocyanine green chorioangiography (ICG) revealed a hypofluorescent choroidal lesion with hyperfluorescent margins (fig. 1b, c). B-scan ultrasonography showed a bilobular choroidal mass with moderate reflectivity, compared to low reflectivity of choroidal melanoma, and measuring $9.5 \times 8.6 \mathrm{~mm}$ with a prominence of $3.9 \mathrm{~mm}$ (fig. 1d). The provisional diagnosis of choroidal metastases or hemangioma was made. Systemic screening for primary tumor was negative. Another session of PDT was performed but the mass did not show any regression on follow-up. Therefore, the decision of performing a biopsy of the lesion was made to establish the definitive diagnosis. Trans pars plana vitrectomy was done and choroidal biopsy was taken.

Histopathology of the obtained sample revealed compact interlacing cords of epithelial cells with fairly large amounts of amorphous extracellular matrix that stained positive with periodic acid-Schiff (PAS) stain (fig. 2a, b). The cells showed no evidence of malignancy. Immunohistochemistry showed that the cells were positive for keratin and negative for Ki67.The final diagnosis of Fuchs' adenoma of the choroid was made.

Postoperatively the lesion was stable. As the condition is benign, no further intervention was made and the patient is on regular follow-up.

\section{Comment}

Benign ciliary epithelioma or age-related hyperplasia of the nonpigmented ciliary epithelium was first described by Fuchs in 1883, and hence called Fuchs' adenoma [6]. Initially it was believed to be a rare tumor, generally found in eyes with a history of ocular inflammation or other abnormalities [7]. But later, Fuchs' adenomas were found incidentally in autopsy series, unrelated to other ocular disorders [2]. Pathologically, the tumor is composed of proliferation of cords of nonpigmented ciliary epithelium embedded in a PAS-positive basement membrane-rich stroma [2]. The tumors are mostly asymptomatic; however, they can mimic the clinical diagnosis of malignancy. So far cases of ciliary body Fuchs' adenoma confirmed on histopathology have been reported but our literature search disclosed no report of a choroidal Fuchs' adenoma [2-4].

In summary, we report a case of Fuchs' adenoma of the choroid presenting as a lesion at the posterior pole and showing no treatment response with PDT. The biopsy confirmed the diagnosis of Fuchs' adenoma. Fuchs' adenoma can occur in the choroid and can simulate hemangioma or malignant melanoma.

\section{Disclosure Statement}

The authors have no financial interests in any aspect of this report. 

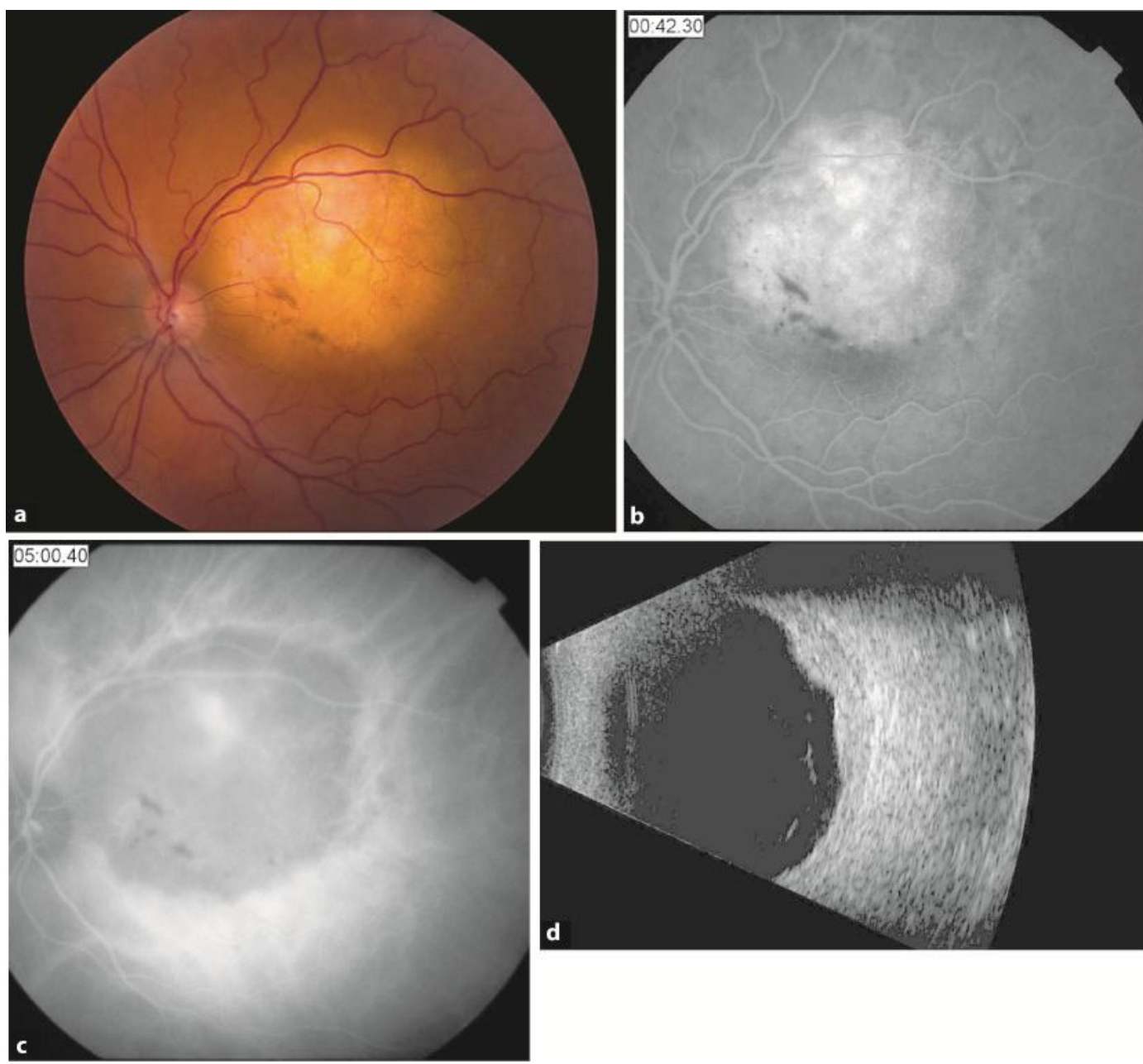

Fig. 1. a Right-eye posterior pole lesion at the time of presentation. $\mathbf{b}$ FFA photograph of the same lesion in venous phase showing hyperfluorescent mass with no pinpoint leakage. c ICG photograph revealed hypofluorescent lesion with hyperfluorescent margins. $\mathbf{d}$ B-scan ultrasonography showed a mid-reflective mass measuring $9.5 \times 8.6 \mathrm{~mm}$ with a prominence of $3.9 \mathrm{~mm}$. 

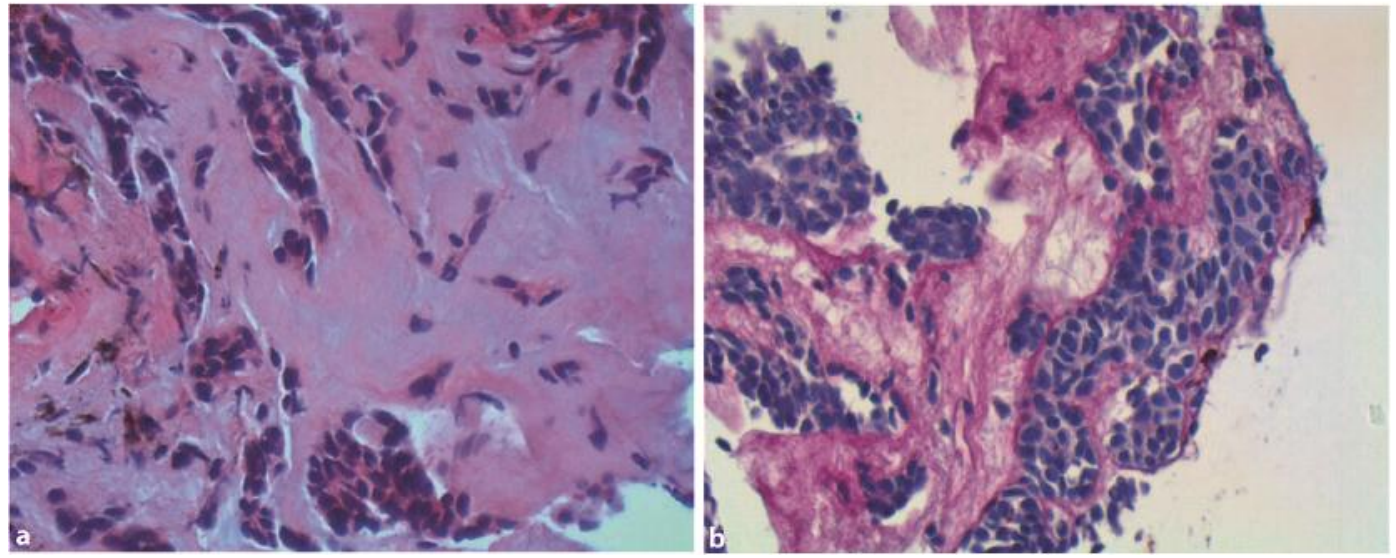

Fig. 2. a Biopsy specimen showing proliferation of epithelial cells with abundant extracellular matrix (hematoxylin-eosin, original magnification $\times 40$ ). $\mathbf{b}$ Same specimen showing PAS-positivity of the extracellular matrix (PAS stain, original magnification $\times 40$ ).

\section{References}

$\checkmark 1$ Zimmerman LE: The remarkable polymorphism of tumours of the ciliary epithelium. Trans Aust Coll Ophthalmol 1970;2:114-125.

-2 Bateman JB, Foos RY: Coronal adenomas. Arch Ophthalmol 1979;97:2379-2384.

3 Zaidman GW, Johnson BL, Salamon SM, Mondino BJ: Fuchs' adenoma affecting the peripheral iris. Arch Ophthalmol 1983;101:771-773.

-4 Shields JA, Shields CL, Eagle RC Jr, Friedman ES, Wheatley HM: Age-related hyperplasia of the nonpigmented ciliary body epithelium (Fuchs adenoma) simulating a ciliary body malignant neoplasm. Arch Ophthalmol 2009;127:1224-1225.

5 Burch PG, Maumenee AE: Iridocyclectomy for benign tumors of the ciliary body. Am J Ophthalmol 1967;63:447-452.

6 Fuchs E: Anatomische Micellen. Albrecht Van Graefes Arch Klin Ophthalmol 1883;29:209-229. Ref Type: Abstract

7 Wolter JR, Pfister RR: Tumors of the pars ciliaris retinae. Am J Ophthalmol 1961;52:659-672. 